\title{
The histomorphological analysis of liver following administration of low doses of diclofenac and ibuprofen
}

\begin{abstract}
This study was conducted to investigate and to compare liver perturbation following administration of low doses of diclofenac and ibuprofen to rats. Hundred and forty-four male Sprague Dawley rats were dosed with 3, 5 and $10 \mathrm{mg} \mathrm{kg-1}$ diclofenac and ibuprofen in saline via intraperitoneal injection for 15 days. The control group was administered with saline in a similar manner. Four rats were euthanised every three days until day 15. Livers were removed, cleaned and a section across the right lobe was taken and fixed in $10 \%$ formalin for light microscopy and TUNEL assay. One-way ANOVA was used to analyse the data. $\mathrm{p}<0.05$ was accepted as significant in this study. Three, 5 and $10 \mathrm{mg} \mathrm{kg}-1$ diclofenac-treated groups and $5,10 \mathrm{mg} \mathrm{kg}-1$ ibuprofen administered groups showed significant changes compared to saline-treated group at day 15 . The changes include presence of focal infiltration by neutrophils and lymphocytes and mild focal necrosis. In 5 and $10 \mathrm{mg} \mathrm{kg}-1$ diclofenac administered groups and $10 \mathrm{mg} \mathrm{kg}-1$ ibuprofen-treated group, apoptotic cells were seen around the perivenular regions (PV) only at day 15 . However, not all the PVs were present with apoptotic cells. This study has shown that, diclofenac is probably more potent in inducing histomorphological changes at low doses. Both the drugs seem to exert time and dose dependent liver morphological alterations to the treated animals.
\end{abstract}

Keyword: Apoptosis; Hepatocytes; Non-steroidal anti-inflammatory drugs 